\title{
Titus Brandsma: Verteidiger der katholischen Presse
}

\author{
$\mathrm{Zu}$ seiner Seligsprechung am 3. November 1985
}

Von Joan Hemels

Schon früher charakterisierte Michael Schmolke Titus Brandsma in einem Beiheft dieser Zeitschrift wie folgt: „Seit 1935 geistlicher Beirat der ,Nederlandsche RoomschKatholieke Journalisten Vereeniging' (N.R.K.J.V. - einem verdienstvollen Journalistenverband), war er (Brandsma) zunächst Innovator, später besonnener Verteidiger der katholischen Publizistik in der Phase der deutschen Besatzung und nationalsozialistischen Unterdrückung."

Obwohl Brandsma gern selbst in Zeitungen und Zeitschriften schrieb, ist es doch bemerkenswert, daß er, der seit 1923 Hochschullehrer an der katholischen Universität in Nijmegen war, seine Zeit und intellektuellen Fähigkeiten in den Dienst der katholischen Journalisten stellte, sei es auf persönlicher Ebene oder im Verband. Der Abstand zwischen Wissenschaft und journalistischer Praxis war vor 50 Jahren noch viel größer als heute. Mitten in einer auch für Journalisten besonderen Zeit der Krise setzte er sich nicht allein für das geistliche Wohl der Journalisten, sondern auch für die Verbesserung ihrer Arbeitsbedingungen und für eine bessere Ausbildung ein. Außerdem beachtete er sehr die Folgen einer neuen Vermittlungstechnik, besonders des Telex, beim nationalen Pressebüro. Er unterstrich für die Journalisten der Tageszeitungen auch die Bedeutung der anderen Medien wie Film, Hörfunk und Fernsehen für die aktuelle Arbeit. Brandsma sah dadurch und unter dem Einfluß der Pressefotografie und der Entwicklung der Publikumszeitschriften eine Verschiebung in der Journalistik auftreten. Nach einer USA-Reise ging der geistliche Berater weit über diese Grenzen hinaus, als er ,seinen' Journalisten Eindrücke von der Entwicklung der Medienlandschaft in den Staaten schilderte. Er war in der Tat - wie Schmolke ihn nennt - ein Innovator.

Dieser Beitrag will Brandsma jedoch vorrangig als jemanden vorstellen, der das Grundrecht des Menschen auf freie Meinungsäußerung innerhalb des Pressewesens verteidigte. Brandsma ließ sich dabei von katholischen Grundsätzen leiten. Entsprechend der damaligen Zeitsituation war die katholische Presse auch für Brandsma ein unentbehrliches Mittel, um den katholischen Glauben und katholische Sitten zu bewahren, zu verteidigen, zu verbreiten und zu vermitteln. Durch seine Stellung als geistlicher Berater der katholischen Journalistenvereinigung wurde Brandsma nach dem deutschen Überfall am 10. Mai 1940 ungewollt zur Schlüsselfigur im Kontakt zwischen den Bischöfen einerseits und den Direktoren und Journalisten der katholischen Presse andererseits. Dies war der Fall im zweiten Jahr der Besetzung, als der Episkopat vor allem in der Person von Erzbischof J. de Jong den katholischen Charakter der noch bestehenden Blätter immer mehr gefährdet sah. Hierin könnte man de Jong mit Kardinal Faulhaber in München vergleichen. De Jong fand in Brandsma einen kundigen und mutigen Berater,

Dr. Joan Hemels ist an der Universität Amsterdam Dozent der Fachgruppe Kommunikationswissenschaften und Massenpsychologie. Sein Lehrauftrag umfaßt die Gebiete Geschichte der Presse, der Propaganda und der öffentlichen Meinung (Kommunikationsgeschichte). 
der bereits vor der Besetzung das volle Vertrauen der katholischen Journalisten gefunden hatte. Gleichzeitig hatte er aber auch durch sein moralisches Gewicht und sein Verhalten das Vertrauen der natürlichen Gegenspieler, der Direktoren der Zeitungsunternehmen.

\section{Bedeutung der Seligsprechung}

Am 3. November 1985 hat Papst Joh. Paul II. in Rom den niederländischen Carmeliterpater Titus Brandsma (1881 - 1942) in das Verzeichnis der Seligen der katholischen Kirche aufgenommen. Diese Seligsprechung nach dreißigjähriger Prüfung des Falles im Prozeß erinnert an einen besonderen Menschen und sein vielseitiges Werk. Kirchenrechtlich bestätigt die Seligsprechung die Überzeugung einer wachsenden Zahl von Personen, daß Brandsma seinen Widerstand gegen die Ideologie des Nationalsozialismus mit dem Märtyrertod bezahlt hat. Mithin wurde er zum Blutzeugen für den christlichen Glauben, als er am 26. Juli 1942 im Konzentrationslager Dachau starb. Nachdem der Papst diese Tatsache des Märtyrertodes bereits am 9. November 1984 offiziell festgestellt hatte, stand der Seligsprechung nichts mehr im Wege.

Seit Papst Urban VIII. (1634) behält sich das Haupt der katholischen Kirche das Recht auf die Seligsprechung selber vor. Im Gegensatz zur Heiligsprechung ist die Seligsprechung weder unfehlbar noch unwiderruflich. Durch das Dekret des Papstes wird den Seligen zuerkannt, als Diener Gottes der himmlischen Glorie teilhaftig zu sein. Im Gegensatz zum Heiligen wird der Selige nicht von der gesamten Kirche öffentlich verehrt, sondern nur an bestimmten Orten.

Seit seiner Gefangennahme durch den deutschen Sicherheitsdienst am 19. Januar 1942 wuchs bei jenen, die ihn näher kannten, die Überzeugung, daß Brandsma mehr war als ein Widerstandsheld. Sie sprachen dies nach dem Zweiten Weltkrieg aus, ohne in kritiklosen Persönlichkeitskult zu verfallen. 40 Jahre nach der Befreiung, im Jahr 1985, gibt es nunmehr in den Niederlanden 35 Straßen und Plätze, die nach Brandsma benannt sind. Schüler von 33 Schulen werden durch den Namen ihrer Ausbildungsstätte an ihn erinnert. In Nijmegen, wo Brandsma seit 1923 wohnte und arbeitete und wo er verhaftet wurde, trägt ein Gemeindehaus und in Colmschate bei Deventer das Pastoralzentrum der dortigen Tituspfarrei seinen Namen.

Der Journalist Louis Frequin, vom 1. Dezember 1945 bis zum 1. September 1977 Chefredakteur der Regionalzeitung „De Gelderlander“, begann die Errichtung einer Stiftung „Gedächtniskapelle Titus Brandsma“. 1959 wurde diese Kapelle mit der künstlerischen Darstellung eines KZ-Wachturms in Nijmegen als ein Platz des Gebets und der Besinnung errichtet. In der gleichen Stadt wird am Todestag Brandsmas seiner in einem eigenen Gottesdienst gedacht. Anläßlich des 40. Jahrestages seines Todes wurde am 24. Oktober 1982 in der Karmeliterkirche St. Maria in Transpontina in der Via della Conciliazione in Rom in einem eigenen Gedächtnisgottesdienst mit Kardinal Alfrink als Zelebrant an ihn gedacht. Einer der Konzelebranten war der frühere Leiter des vatikanischen Pressesaales, Mgr. Fausto Vallainc, jetzt Bischof von Alba. Dieser Bischof schrieb ein Buch über Titus Brandsma unter dem Titel „Un Giornalista martire, Padre Tito Brandsma“, das 1984 in zweiter Auflage erschien. ${ }^{2}$ Eine Woche nach der Seligsprechung in Rom fand am 10. November 1985 die nationale Feier dieses Ereignisses in der St. Jan-Kirche in Den Bosch statt. In dieser eben voll restaurierten gotischen Kathedrale wurde der Selige am 17. Juni 1905 zum Priester geweiht. 
Durch Studientage, Zeitungs- und Zeitschriftenartikel und eine Fernsehdokumentation, die am 14. November 1985 ausgestrahlt wurde, sowie durch ein inzwischen auch in deutscher Sprache veröffentlichtes Buch ${ }^{3}$, wurde die Gestalt von Titus Brandsma in den Medien sehr beachtet. Zusammenfassend kann man feststellen, daß der Papst den Niederlanden keinen Seligen aufgedrängt hat. Brandsma hat vielmehr als Opfer des Naziterrors und der antichristlichen nazistischen Ideologie ${ }^{4}$ für den modernen Menschen Symbolcharakter. Es wird sorgfältig darüber gewacht, keine ungesunden nationalen Gefühle zu wecken und man ist bemüht, einen unangemessenen Personenkult zu vermeiden. In einer pluriformen Gesellschaft wie den Niederlanden mit einer außerdem auf Konservative, Progressive und Mittelkurs verteilten katholischen Glaubensgemeinschaft, ist eine Seligsprechung eine delikate Angelegenheit, die auch in den Medien leicht mißverstanden werden kann.

\section{Der Widerstand von Titus Brandsma}

Schon 1973, als das öffentliche Interesse an einer Seligsprechung noch sehr gering war, veröffentlichte "Communicatio Socialis" einen Artikel über die Bedeutung Brandsmas für die katholische Presse in den Jahren 1935 bis $1942^{5}$. Die Aufgabe dieses Beitrages war es damals nicht, die mühsame Arbeit der Kongregation für die Selig- und Heiligsprechungen im Vatikan zu unterstützen. Von Anfang an hat der Vizepostulator im Seligsprechungsprozeß, der niederländische Karmelit Dr. A. Staring, klargestellt, daß es sich bei Brandsma um ein „Martyrium ex aerumnis carceris“, also um ein Martyrium durch die Entbehrungen der Gefangenschaft handle. Als der Artikel in „Communicatio Socialis" erschien, war der damalige Promotor Fidei, welcher die vorgebrachten Argumente für die Seligsprechung hinterfragen muß, noch nicht davon überzeugt, daß Brandsma als Märtyrer des Glaubens gestorben sei. Dadurch verzögerte sich der Prozeß. Es mußte bewiesen werden, daß Brandsma mehr war als nur ein Widerstandsheld aus dem Zweiten Weltkrieg.

Bei der Beurteilung des Widerstandes in der niederländischen Besatzungszeit muß man zwischen paramilitärischem (nationalem) und ethischem oder bürgerlichem Widerstand unterscheiden. Dieser letztgenannte Widerstand etwa der Untergrundpresse und der Kirchen war darauf gerichtet, die Gleichschaltung des gesellschaftlichen Lebens im nationalsozialistischen Sinn zu verhindern. Es gibt keinen Zweifel, daß Brandsma dabei einen wesentlichen Anteil hatte. Darum wurde ihm posthum am 5. Mai 1982 das 1980 gestiftete Widerstandsgedächtniskreuz zuerkannt. In einem an die Familienmitglieder Brandsmas gerichteten Brief vom 4. April 1946 hatte schon Königin Wilhelmina der Niederlande über „diesen großen und aufrechten Patrioten" geschrieben. Er hat - so die Königin in ihrem Brief - nicht gezögert, allen, die ihn um Rat fragten, von Anfang an deutlich den religiösen und vaterländischen Standpunkt vor Augen zu führen. Auch als er schon im Gefängnis war, hörte er nicht auf, seinen Standpunkt über die nationalsozialistische Lebensanschauung zu Papier zu bringen ${ }^{6}$.

Das Standardwerk über das Königreich der Niederlande im Zweiten Weltkrieg enthält im zweiten Band des fünften Teils einen Text von rund 12 Seiten mit einem Bild von Titus Brandsma.' Der Autor de Jong schreibt mit viel Wohlwollen über Brandsma und seine Bedeutung für den Aufbau einer Einheitsfront der katholischen Presse gegen die Zwangsmaßnahmen der Besatzer. Ende 1941 und Anfang 1942 bedeutete dies konkret, daß Brandsma in enger Überlegung mit dem Erzbischof de Jong eine schriftliche 
Erklärung der katholischen Tageszeitungen verlangte, keine Anzeigen der nationalsozialistischen Bewegung abzudrucken. Im Jahre 1935 hatte der damalige Erzbischof J.H.G. Jansen Brandsma im Namen des Gesamtepiskopates zum geistlichen Berater der Vereinigung katholischer Journalisten ernannt. Die Beziehung zwischen Episkopat und katholischer Presse wurde auch in der Tatsache deutlich, daß nur jene Zeitungen als katholisch angesehen wurden, die einen vom Bischof ernannten Zensor hatten. Brandsma war 1938 zum kirchlichen Zensor des „De Gelderlander“ in Nijmegen ernannt worden.

1941 wollten die Besatzer die katholischen Tageszeitungen zwingen, mit Anzeigen und redaktionellen Beiträgen für Organisationen zu werben, in denen die Mitgliedschaft von Katholiken durch die Bischöfe verboten war. Dabei ging es besonders um die „Opvoedersgilde", eine Organisation der nationalsozialistischen Lehrer und Erzieher. Auf ausdrücklichen Rat von Brandsma verbot Erzbischof de Jong den katholischen Zeitungen, die umstrittenen Anzeigen zu veröffentlichen. Brandsma bot sich außerdem freiwillig an, die Direktoren und Chefredakteure der katholischen Tageszeitungen zu besuchen und ihnen ein Exemplar eines von ihm geschriebenen Briefes vom 31. Dezember $1941^{8}$ zu überbringen und mündlich zu erläutern. Gleichzeitig wollte er auch die anderen Bischöfe informieren.

Am 2. Januar 1942 begann Brandsma seine Rundreise zu den katholischen Zeitungen. Einige der für katholische Zeitungen verantwortlichen Personen, mit denen Brandsma sprach, erbaten von ihm ein offizielles Schreiben der Bischöfe. Es stellte sich heraus, daß alle Bischöfe sich dem Standpunkt des Erzbischofs anschließen würden. Der Erzbischof verfaßte deshalb auf Grundlage eines Konzeptes von Brandsma eine Instruktion für die Direktoren und Chefredakteure der katholischen Tageszeitungen. Alle Bischöfe waren mit dem Inhalt und seiner Darstellung einverstanden. Anzeigen der Nationalsozialistischen Bewegung (NSB) sollten von katholischen Zeitungen nicht angenommen werden. Bei Zuwiderhandlung drohte den Verantwortlichen die Verweigerung des Sakramentenempfanges. Berichte und Nachrichten von NS-Seite, deren Aufnahme verpflichtend war, mußten klar mit der entsprechenden Quellenangabe versehen werden. Bevor dieser Brief mit Instruktion am 16. Januar $1942^{9}$ verschickt wurde, hatten die Besatzer bereits beschlossen, Brandsma „wegen planmäßiger Vorbereitung einer gegen die deutschen Besatzungsbehörden gerichteten oppositionellen Bewegung umgehend zu verhaften und einem Konzentrationslager zuzuführen ${ }^{* 10}$. Durch die Mitarbeit des Direktors der katholischen Tageszeitung des Südens (,Dagblad van het Zuiden“), Hubertus Vervoort ${ }^{11}$, war die Abteilung Presse des Ministeriums für Volksaufklärung und Künste in Den Haag bereits am 5. Januar 1942, unmittelbar nach Brandsmas Besuch bei dieser Zeitung, über die Gründe für Brandsmas Rundreise informiert worden. Am 6. Januar 1942 holte ein Beamter dieser Abteilung, H.A. Goedhart, in Eindhoven eine Kopie des Rundschreibens von Brandsma ab. Tragisch ist es, daß der antideutsch eingestellte Vater des für den Verrat verantwortlich gehaltenen Zeitungsdirektors in Eindhoven, Adriaan J.M. Vervoort, am 8. Januar nach Nijmegen reiste. Er warnte dort den Prior des Karmelitenklosters und suchte ihn zu bewegen, Brandsma untertauchen zu lassen. Brandsma weigerte sich, verbrachte aber einige Tage in einem anderen Kloster des Ordens, um sich zu erholen. Am 19. Januar 1942 wurde er in seinem Kloster in Nijmegen verhaftet. 


\section{Die Haltung des Episkopates}

Schon 1934 warnte der niederländische Episkopat ernsthaft vor der nationalsozialistischen Bewegung (NSB) in den Niederlanden. Im Jahre 1936 fügte er hinzu, daß jene, die diese politische Partei in größerem Maße unterstützten, von den Sakramenten ausgeschlossen werden müßten. Am 13. Januar 1941 wurden diese Maßnahmen auf alle ausgedehnt, die Mitglieder der NSB waren oder eine ihrer Schwesterorganisationen stärker unterstützten. Am 25. Juli 1941 verbot der Episkopat unter strenger Exkommunikation auch die Mitgliedschaft in den Schwesterorganisationen. Mit Blick auf diese Entwicklung holte der Erzbisch of von Brandsma eine Stellungnahme über den Verband Niederländischer Journalisten ein, der am 24. August 1940 gegründet worden war. Inzwischen gehörten ihm auch die noch in ihren Berufen tätigen katholischen Journalisten an. In einer ausführlichen Stellungnahme vom 23. September 1941 kam Brandsma, die Argumente für und wider sorgfältig abwägend, zu folgender Schlußfolgerung: „Primär an der Forderung, daß die Katholiken den ,Verbond" verlassen sollen, ist das Verlangen, auf eigene Tageszeitungen zu verzichten, die jetzt noch bestehen. Die Umstände erfordern es meiner Meinung nach noch nicht, diese letzte Forderung zu stellen. Deshalb ist es meines Erachtens gerechtfertigt, daß die Katholiken noch Mitglied im ,Verbond bleiben" ${ }^{* 12}$.

Noch vor Ende des Jahres 1941 war wegen der Anzeigen und des dabei erfolgten Zwangs sowohl bei Brandsma als auch beim Erzbischof und - wie sich bald zeigte - auch bei den anderen Bischöfen das $\mathrm{Ma} ß$ voll. Nicht aus politischen, sondern aus prinzipiellen Gründen nahmen sie eine unerschütterliche Haltung ein. In dem Brief Brandsmas vom 31. Dezember 1941 ist dann auch die Rede von folgender Stellungnahme: „Seit einigen Tagen ist durch leitende Instanzen der Tagespresse eine Anweisung gegeben worden, deren Befolgung die Leitungen und Redaktionen katholischer Zeitungen in Gegensatz bringt zu den eigenen Prinzipien. Ausdrücklich ist in dieser Weisung, die die Aufnahme von Anzeigen der nationalsozialistischen Bewegung (NSB) zur Pflicht macht, gesagt, daß man sich ihr nicht aus Gewissensgründen widersetzen darf. Hier wird durch die Führung das Gewissen selbst zum Diskussionspunkt gemacht. Hier können die katholischen Tageszeitungen nicht mehr nachgeben, wenn sie nicht in Gegensatz zur Weisung ihres Bischofs treten wollen, der ihnen verbietet, der Bewegung bedeutende Hilfe zu leisten." ${ }^{\text {"13 }}$ Die Grenze war erreicht, das stand Anfang 1942 fest. An der Meinungsbildung und der Beurteilung des Episkopates hatte Brandsma ausschlaggebenden Anteil. Die Frage, ob bis dahin der Episkopat und Brandsma eine langmütige oder abwartende Stellung einnahmen, kann meiner Ansicht nach nicht positiv beantwortet werden. Doch wird dies in der notwendigen Beurteilung der Journalistik der deutschen Besatzungszeit wohl immer eine Streitfrage bleiben. Das Gleiche gilt für die Frage, wer mehr Verantwortung für den Fortbestand der katholischen Presse in der Besatzungszeit trägt, die Bischöfe oder die Eigentümer der katholischen Blätter. Ich persönlich meine, daß die Letztgenannten die Endverantwortung auch für den Inhalt ihrer Blätter hatten.

\section{Absicht der Besatzer}

Vor seiner Ankunft am 19. Juni 1942 in Dachau verbrachte Brandsma fast ein halbes Jahr in verschiedenen Gefängnissen. Da die Besatzer in den Niederlanden nicht befugt waren, Gefangene unmittelbar ins Konzentrationslager zu schicken, mußte das Reichssicherheitshauptamt in Berlin einen Schutzhaftbefehl ausstellen. Dafür mußte der 
Bericht der Sicherheitspolizei nach Berlin geschickt werden, der dann mit den bereits über Brandsma vorliegenden Gegebenheiten über seinen Kampf gegen den Nationalsozialismus und gegen den Antisemitismus verglichen wurden. ${ }^{14}$

Am 21. Januar 1942 wurde Brandsma zum ersten Mal vom Befehlshaber des Sicherheitsdienstes in Den Haag verhört. Die Schlußfolgerung war: „Brandsma und der Erzbischof de Jong sind also die treibenden Kräfte, die unsere Bestrebung, eine deutsche Beeinflussung des niederländischen Volkes durch die Presse, sabotieren. Die Maßnahmen der deutschen Behörden werden durch die Wühlarbeit des Professor Brandsma erheblich gestört. Er ist ein gefährlicher Mensch. Seine Tätigkeit ist dazu geeignet, das Ansehen des Deutschen Reiches und des Nationalsozialismus zu bekämpfen und die Einheit des niederländischen Volkes zu untergraben. Eine längere Inschutzhaftnahme Professor Titus Brandsmas erscheint angebracht. ${ }^{\text {c15 }}$ Am 6. Mai 1942 erfolgte ein zweites Verhör. Als dabei deutlich wurde, daß Brandsma seine Ansichten nicht geändert hatte, wurde ihm mitgeteilt, daß er bis zum Ende des Krieges im Konzentrationslager Dachau bleiben müsse. Im B lick auf die Gesundheit von Brandsma und die Zustände in Dachau bedeutete dies ein Todesurteil auf Raten.

Aus Brandsmas Schriften selbst, aus dem Verhörprotokoll und dem Bericht über seinen Fall, der am 22. Januar 1942 nach Berlin geschickt wurde, wird deutlich, daß Brandsma wegen seiner Aktion für die katholische Presse verhaftet und wegen seiner Verteidigung katholischer Grundsätze verurteilt wurde. Die Art und Weise, wie er sich schon früher gegen den Antisemitismus geäußert hatte und seine Verteidigung des katholischen Unterrichts gegenüber der Besatzungsmacht können eine zusätzliche Rolle gespielt haben.

In der Person von Brandsma wollte das Ministerium für Volksaufklärung und Künste im Zusammenspiel mit dem Reichskommissariat Niederlande und seinen Unterabteilungen wahrscheinlich auch symbolisch den Episkopat strafen. „Die Haltung des niederländischen Episkopates ist auch die meine," hatte Brandsma in seinem Verhör gesagt.

Obwohl Brandsma sich in seinen vielen Aktivitäten nicht mit Politik im strengen Sinn beschäftigte, sahen die Nationalsozialisten in der Verteidigung kirchlicher Grundsätze und kirchlicher Verbundenheit der Presse und Erziehung einen ,politischen Katholizismus". Brandsma protestierte dagegen und wollte auch nicht als ,deutschfeindlich" abgestempelt werden.

Nach der Verhaftung von Brandsma gab es keine weiteren Verhaftungen. Auch Erzbisch of de Jong blieb ungeschoren, wahrscheinlich weil die Besatzer die Folgen fürchteten, die eine Festnahme dieses geistlichen Leiters sowohl bei Katholiken als auch bei anderen Christen haben würde. Außerdem wurden die Anzeigen der NSB, die zu der harten Konfrontation geführt hatten, zurückgezogen. Die NSB blieb die einzige zugelassene Partei. Als solche wurde sie am 14. Dezember 1941 durch den Reichskommissar für die besetzten niederländischen Gebiete, Dr. Arthur Seyss-Inquart, ausgewiesen, und dies hatte zur Folge, daß die niederländische Presse grundsätzlich die Aufnahme von NSB-Anzeigen nicht verweigern konnte. Da aber die NSB durch den Episkopat zu einer verbotenen Organisation erklärt worden war, entstand ein Gewissenskonflikt für die katholische Presse. 


\section{Gleichschaltung statt Aufhebung}

Am 7. Januar 1942 schrieb Pressedezernent Willy Janke eine Notiz für den Generalkommissar zur besonderen Verwendung im Reichskommissariat Niederlande, Fritz Schmidt („Betr. Beabsichtigte Obstruktionsaktion der katholischen Presse“). Darin steht etwas, das nicht zu kontrollieren war und vor dem die Besatzer offensichtlich Angst hatten. Es geht um das, was in diesem Text „,der Tatbestand“"genannt wurde. Es heißt dort: „Wie ich soeben erfahre, ist dieser Tage auf Betreiben der katholischen Bischöfe eine Aktion unter der katholischen Presse der Niederlande zu dem Zweck gestartet worden, alle katholischen Blätter ,aus Protest gegen bestimmte Anweisungen der niederländischen Obrigkeit' einstellen zu lassen ... Herr Titus Brandsma soll in seinen Unterredungen betont haben, wenn die Direktionen und Hauptschriftleitungen der verschiedenen katholischen Blätter mit diesem Plan einverstanden wären, würde Herr Boerrigter von ,De Tijd' in ultimativer Form vom Leiter der Presseabteilung, Herrn Janke, die Auflösung der gesamten katholischen Presse in den Niederlanden fordern. Bemerkenswert ist noch, daß die Bischöfe für den Fall des Mißlingens der von Herrn Titus Brandsma geleiteten Aktion die Absicht haben sollen, die katholische Bevölkerung von der Kanzel herab zur Aufkündigung sämtlicher Abonnements der katholischen Zeitungen aufzufordern (!).616

So weit ist es nicht gekommen. Die Besatzer wollten die katholische Presse nicht verschwinden lassen, sondern gleichschalten. Auch Erzbischof de Jong war Anfang 1942 nicht bereit, die noch bestehenden 17 katholischen Tageszeitungen durch die Deutschen schließen zu lassen. In diesem Zusammenhang schrieb er seinen Mitbischöfen am 15. Januar 1942, daß eine Schließung nicht nur einen gewaltigen wirtschaftlichen Schaden für viele, die bei der katholischen Presse arbeiteten, bedeute, sondern auch einer Schädigung der Eigentümer und Direktoren gleichkomme. Es würde auch bedeuten, daß jene Katholiken, die trotzdem eine Zeitung lesen wollten - und wenn nur wegen der Anzeigen - gezwungen würden, die „gefährlich neutrale Presse“ zu beziehen. „Eine katholische Zeitung, die noch eben bestehen kann, ist besser als eine neutrale“, ließ der Erzbischof seine Mitbischöfe wissen. ${ }^{17}$

In ihrem Hirtenbrief vom 25. Juli 1941 hatten die Bischöfe schon mit diesem Satz die Lage geschildert, in die die katholische Tagespresse nach gut einem Jahr deutscher Besatzung geraten war: „Unsere katholische Tagespresse ist entweder aufgehoben oder so in ihrer Freiheit begrenzt, da 3 man praktisch von katholischen Zeitungen nicht mehr sprechen kann.“'18 Auf diesem Hintergrund muß man das Auftreten von Erzbischof de Jong und Brandsma im Herbst und Winter 1941/42 sehen. Auch in anderen Bereichen begann damals der gut organisierte Widerstand.

\section{Die Motivation von Titus Brandsma}

Auf die Frage, warum Brandsma trotz seines Wissens um das damit verbundene Risiko sich bis zum Äußersten für die Sache der katholischen Presse eingesetzt hat, erhält man meiner Meinung nach ein klare Antwort in dem folgenden Abschnitt der BrandsmaBiographie von Dölle: „Titus ging in den großen Streit um der Eigenverantwortung der Presse und der Freiheit des Wortes willen. Für ihn war es eine Sache der Gerechtigkeit, die immer im Mittelpunkt messianischer Erwartung gestanden hat und die nicht durch Gesetze blockiert werden kann. Für diesen Streit braucht man Mut, einen Mut, der 
einen zum Leichtsinn bis an die Grenze führen kann, wo die Gewalt heimtückisch wird und sich verhärtet. In einem solchen Streit auf Leben und Tod gibt es schnell eine kriegerische Sprache, die zu nichts verpflichtet. Aber Titus war nicht nur ein Mann der Worte, er ging dem Streit nicht aus dem Wege, der schließlich alles von ihm forderte, was ein Mensch geben kann. “19

Oben wurde bereits angemerkt, daß Brandsma die Rolle und Aufgabe einer katholischen Presse so sah, wie sie vor einem halben Jahrhundert in katholischen Kreisen der Niederlande allgemein betrachtet wurden. Er entwickelte deswegen keine neue Theorie der katholischen Presse, aber gab eine Synthese dessen, was Päpste und Bischöfe dazu gesagt hatten. Wenn die Presse keine „arma veritatis“ mehr ist, das Ideal einer starken Waffe im Streit für die Wahrheit, dann hat sie auch ihren Wert für die Kirche verloren. Entsprechend den damals vorherrschenden Gedankengängen war Brandsma überzeugt vom Einfluß der Presse zum Guten und zum Bösen. „Eine Zeitung“, so sagte er 1939 in einer Ansprache beim katholischen Rundfunk (KRO), ,ist wie ein Freund, dessen Urteil man schätzt, dem man gerne zuhört, dessen Vorschläge man nicht übergeht. Ein altes französisches Sprichwort sagt: Sag mir, mit wem du umgehst, und ich sage dir, wer du bist. Das gilt auch für die Presse. An der Zeitung erkennt man den Leser; denn er wählt seine Zeitung, er liest, was er lesen will. Er stellt sich unter einen Einfluß, dem er nicht entrinnt." Brandsma erkannte, daß die nicht aus katholischer Gesinnung redigierten Zeitungen in bestimmten Punkten den katholischen Zeitungen in ihrer Qualität nicht gleichkämen. Dabei stellte er sich nicht auf den fixen Standpunkt, sie seien voller Unwahrheiten und irreführender Standpunkte. Im Gegenteil: seiner Ansicht nach dominierten die guten Beiträge auch in nationalsozialistischen Zeitungen. Aber es gebe halt auch irreführende und gefährliche Vorstellungen, die immer wieder eingestreut seien. Diese Zeitungen, so sagte Brandsma seinen Zuhörern, seien nun einmal ein Sprachrohr von Menschen, die in sehr wichtigen Dingen anders denken und glauben als wir. Das kommt, wenn auch nicht täglich und in jedem Blatt, aber doch regelmäßig vor. Der regelmäßige Leser kann sich diesem Einfluß nicht entziehen. ${ }^{20}$

Aber außer dem ideellen Charakter anerkannte Brandsma auch die wirtschaftliche Seite des Zeitungsunternehmens, der er den ganzen zweiten Teil seines Vortrages widmete. ${ }^{21}$ In Verbindung mit seiner unverrückbaren Haltung in der Frage der NSB-Anzeigen und der NSB-Berichterstattung war es wesentlich festzustellen, daß Brandsma von dem verderblichen Einfluß der nationalsozialistischen Tendenzen der Tagespresse überzeugt war. Mit der Auflösung katholischer Tageszeitungen sah er das Ende einer positiven Einflußmöglichkeit der Kirche gekommen.

Auf Wunsch seines Richters, des Verantwortlichen des Sicherheitsdienstes in Den Haag, legte Brandsma im Gefängnis schriftlich dar, warum das niederländische Volk und vor allem der katholische Volksteil gegen die nationalsozialistische Bewegung war. Unerschrocken, aber ohne auch nur einen Anflug von Haß, stellte Brandsma dar, wie die betreffende Bewegung durch antinationale und vor allem auch antichristliche Tendenzen gekennzeichnet sei. Er endete seine Darlegung mit einer Bitte an Gott für Deutschland mit folgenden Worten: „Gott segne die Niederlande, Gott segne Deutschland. Gott gebe, daß beide Völker bald wieder in vollem Frieden und Freiheit nebeneinander stehen in Seiner Erkenntnis und zu Seiner Ehre, zum Heil und zur Blüte beider so verwandten Völker.“22 Wer diesen Friedenswunsch von Brandsma, der durch einen Mitbruder mit Recht ein ,Friedensprophet ${ }^{`}$ genannt wurde ${ }^{23}$, auf sich wirken läßt spürt, daß die Seligsprechung auch dem Verhältnis zwischen den Niederlanden und Deutschland zugute kommt. Seine Friedensbotschaft stiftet noch vierzig Jahre nach dem Zweiten Weltkrieg Versöhnung. 
Für beide Länder gilt auch heute noch, daß die Pressefreiheit ,zu den großen Ideen der Menschheitsgeschichte und ihre Durchsetzung zu jenen Errungenschaften, die eine Grundvoraussetzung bilden für die heutige Existenz freiheitlicher, demokratisch verfaßter Gesellschaften und politischer Systeme ${ }^{\text {(24 }}$ gehört. Die Zeit des Nationalsozialismus ließ weder in Deutschland noch in den Niederlanden Raum für eine juristische Diskussion der Pressefreiheit. Brandsma verwies hier auf den Bereich des ethisch-religiösen Denkens und Handelns und gab damit der Diskussion über die Pressefreiheit eine neue Dimension.

\section{Anmerkungen}

1 Michael Schmolke: Pluralistische Publizistikstrukturen und ihre Probleme. In: Joan Hemels und Michael Schmolke (Hrsg.): Katholische Publizistik in den Niederlanden. München-Paderborn-Wien 1977, 7-14, hier 9.

2 Milano, Editrice Ancora. Vgl. Gino Concetti: Il sacrificio di Tito Brandsma. In: LOsservatore Romano vom 1. Mai 1985.

3 Constant Dölle: Titus Brandsma - Karmeliet. Amstelveen 1985.

4 Laut Bernardus Kardinal Alfrink in seiner Predigt am 24. Oktober 1982 in Rom, im Wortlaut abgedruckt in der Informationsbroschüre der Stiftung Gedächtniskapelle Titus Brandsma, Ausgabe Herbst 1983 (Titus Brandsma Fonds, Karmelitenkloster, Steenstraat 39, NL-5831 JA Boxmeer, wo sich auch das Titus Brandsma Archiv befindet - seit 1980).

5 Joan Hemels: Titus Brandsma und die katholische Presse der Niederlande zwischen 1935 und 1942. In: CS 6:1973, 1-26. Auch erschienen in Hemels-Schmolke, a.a.O., 15-38.

6 Brief in Wortlaut in Brocardus Meijer: Titus Brandsma. Bussum 1951, 464. G.R. Zondergeld: Brandsma, Anno Sjoerd. In: J. Charité (Endredaktion): Biographisch woordenboek von Nederland, Band 1. 's-Gravenhage 1979, 85-86, charakterisiert Brandsma in diesem neutralen biographischen Nachschlagewerk, 86, als Märtyrer für Land und Glaube und skizziert in Kürze dessen Bemühen für die katholische Presse aus Anlaß seines Todes.

7 L. de Jong: Het Koninkrijk der Nederlanden in de Tweede Wereldoorlog. 5. Teil: Maart '41-juli '42, zweiter Band. 's-Gravenhage 1974, 747-759. De Jong nahm keine Kenntnis von der in Anmerkung 4 genannten Studie und verfügte über weniger Unterlagen. Trotzdem schreibt er a.a.O., 749, ohne es zu belegen: ,A uch seinen Gutachten gemäß hatte dieser Verein (d.h. die Vereinigung niederländischer katholischer Journalisten N.R.K.J.V.) sich 1940 kaum der Gleichschaltung widersetzt.' Meiner Meinung nach ist diese Behauptung anfechtbar. Vgl. die Ergebnisse meiner Forschungsarbeit, erwähnt in Anmerkung 4, 7-12 bzw. 21-25. Außerdem blieb die N.R.K.J.V mit einer besonderen Aufgabe der Vertretung der spezifischen katholischen Belange bis Juli 1942 weiter bestehen. In einem Gutachten an den Erzbischof vom 23. September 1941 (a.a.O., 10-12 bzw. 24-25) machte Brandsma eine Abwägung, die vom Episkopat übernommen wurde, und sie bietet meines Erachtens noch keinen Grund zu einem Vorwurf eines Mangels an Widerstandsbereitschaft.

8 Niederländische und deutsche Fassung dieses Briefes in Hemels, a.a.0., 16-19 oder HemelsSchmolke, 30-32.

9 ebda., 19-20 oder 32-33.

10 Faksimile der ,Aufzeichnung für Herrn Generalkommissar Schmidt' des Leiters der PresseAbteilung' (W. Janke) mit dem Vorschlag, Brandsma zu verhaften usw. In: De Jong: a.a.O., neben 753 .

11 Vgl. die Charakterisierung dieses Zeitungsdirektors und dessen Tageszeitung in S. Zoetmulder; Welk doel heiligde welke middelen? Veertig jaar geleden in de Brabantse perswereld. In: Brabantia,'s-Hertogenbosch 31: 1982, Nr. 2, 49-58. Hub. Vervoort weigerte sich als Verantwortlicher für die de iure noch katholische, de facto jedoch dem Nationalsozialismus positiv gegenüberstehende 'Tageszeitung Dagblad von het Zuiden in Eindhoven, Brandsmas Gedankengut und - 
später - die bischöflichen Richtlinien zu befolgen. Zoetmulder ist davon überzeugt, daß die Kurie des Bistums 's-Hertogenbosch Brandsma bei seinem Besuch am 3. Januar 1942 hätte warnen können. - Dagblad van het Zuiden war seit dem 3. November 1941 die Fortsetzung der katholischen Eindhovensche en Meierijsche Courant; es gab keine Fusion mit Eindhovensche Dagblad, wie ich fälschlicherweise in Hemels, a.a.O., 24, Anm. 44 schrieb. Letztgenannte Tageszeitung wollte sich nicht mit einer opportunistischen Zeitung verschmelzen und erschien deshalb am 31. Oktober 1941 zum letzten Mal. - Anfang 1942 verfügte Dagblad von het Zuiden noch immer über einen von dem Bischof von's-Hertogenbosch ernannten Censor; die Zeitung war auf Grund dieser Tatsache eine katholische Tageszeitung und deshalb mußte m.E. Brandsma auch Hub. Vervoort besuchen.

12 Vgl. Hemels, a.a.O., 12 oder Hemels-Schmolke, 25.

13 ebda., $17-18$ und 31 .

14 De Jong, a.a.O., 755.

15 Zitat in Godfried Bomans: Prof. Dr. T. Brandsma, O. Carm. In piam memoriam. In: Elseviers Weekblad, Amsterdam 2:1946, Nr. 38, 1.

16 Faksimile in De Jong, a.a.O., gegenüber 753.

17 Diese Erläuterung wurde (vgl. De Jong, a.a.O., 753 und Hemels, a.a.O., 14 oder Hemels-Schmol$k e, 28)$ am 15. Januar 1942 von Erzbischof De Jong den anderen Bischöfen mitgeteilt zur Begründung der folgenden Ergänzung: ,Bei der zur Pflicht gemachten Veröffentlichung von Nachrichten und Berichten von nationalsozialistischer Seite muß die Quelle angegeben werden'. Die Erläuterung wurde nicht, wie De Jong, a.a.O., 753 suggeriert, im Schreiben des Episkopats vom 16. Januar 1942 hineingefügt.

18 Hirtenbrief in S. Stokman: Het verzet van de Nederlandsche Bisschoppen tegen nationaalsocialisme en Duitsche tyrannie. Herderlijke brieven, instructies en andere documenten. Utrecht $1945,200$.

19 Dölle, a.a.O., 40.

20 Die Hörfunkansprache vom 29. Januar 1939, als ,Stelle und Aufgabe einer eigenen katholischen Presse' tituliert, wurde in zwei Teilen in der Tageszeitung Ons Noorden vom 11. und 14. Januar 1939 veröffentlicht. Das Zitat wurde dem ersten Teil entnommen.

$21 \mathrm{Vgl}$. Ons Noorden vom 14. Januar 1939.

22 Stellingen en artikelen voor te leggen in de zaak der zaligen heiligverklaring van de Dienaar Gods Titus Brandsma, priester van de Orde der Broeders van O.L. Vrouw van de Berg Carmel, (Nijmegen) 1955, 26.

23 In dem Aufsatz von Hein Blommestijn: Titus Brandsma: Vredesprofeet en oorlogsmartelaar. In: Speling, 36:1984, Nr. 4, 42-49.

24 Jürgen Wilke (Hrsg.): Pressefreiheit. (Einleitung). Darmstadt 1984, 1-57, hier 1. 


\section{SUMMARY}

The beafication of the carmelite Titus Brandsma (1881-1942) on the 3rd of November 1985 leads the author to at least two essential questions. First: what's the importance of his activities for the catholic press in the Netherlands under the German occupation? Second: what's the meaning of his martyrdom for the freedom of the press in our time? Basing his examination on his former study, published in CS 6/1973, nr. 1, p. 1-25, the author weighs the cardinal role of Brandsma in coming to decisions on a clear standpoint against the press politics of the German authorities and their Dutch collaborators, after the invasion of 10th May 1940. Journalists and directors of catholic newspapers, developed - sometimes with overt or silent support of clerical advisers - divergent views and practices, with the risk of steadily going further in making compromises. In the opinion of Brandsma this dangerous attitude was necessarily a lead to commit themselves. He persuaded the Dutch bishops, at the end of 1941 , to reject absolutely new measures of the occupying powers who tried to use the press for propagating the national-socialistic ideology. Brandsma did not argue on a small level of ecclesiastical law or on a constitutional basic, he gave the fundamental discussion on freedom and responsibility of the press a new ethical-religious dimension. In doing this he was giving a message for us in our time. Being also a prophet of peace he was praying and pleading before his martyrdom for reconciliation between the peoples of Germany and the Netherlands, both liberated from Nazism.

\section{RÉSUMÉ}

A l'occasion de la béatification du Carmélite Titus Brandsma (1881-1942) qui aura lieu le 3 novembre 1985, l'auteur se pose deux questions essentielles. Quelle est l'importance de ces activités en faveur de la presse catholique néerlandaise pendant l'occupation Allemande? Quelle est la signification de son martyr pour la liberté de la presse aujourd'hui? En basant son étude sur un article antérieur, publiée dans C.S. 6/1973, No. 1, p.1-25, l'auteur accorde un rôle important à Brandsma qui, après l'invasion du 10 mai 1940, pris une position fermement opposée à la politique des autorités Allemandes et de leurs collaborateurs néerlandais en matiere de presse. Des journalistes et des directeurs de journaux catholiques developpaient - quelque fois avec le support ouvert ou caché de conseillers religieux - des vues et practiques divergantes avec le risque de compromissions avec l'occupant. Pour Brandsma, ils ne pouvaient que tomber dans une attitude de collaboration. Vers la fin de 1941, Brandsma persuada les Evêques hollandais de refuser avec détermination toutes nouvelles mesures imposées par les forces d'occupation pour la propagation de l'idéologie nationale-socialiste. Il ne maintint pas le débat au seul plan de droit ecclésiastique, ni au niveau constitutionnel, mais il donna une dimension éthico-religieuse nouvelle aux questions fondamentales de la liberté et de la responsibilité de la presse. En cela, il laisse un message, qui, pour nous, reste actuel. En prophèt de la paix, il pria et plaida, avant même son martyr, pour que les peuples de l'Allemagne et des Pays-Bas se réconcilient après leur libération mutuelle du nazisme.

\section{RESUMEN}

Según el autor dos preguntas esenciales se presentan con ocasión de la beatificación del Carmelita Titus Brandsma (1881-1942), que se realizará en el dia 3 de Noviembre 1985. Cuál es la importancia de sus actividades en la prensa católica holandesa durante la ocupación alemana? ... Cuál es la significación de su martirio para la libertad de la prensa en nuestros tiempos? Basando su análisis sobre un estudio anterior, publicade en Communicatio Socialis, 6/1973, no. 1, p. 1-25, el autor sen̂ala el importante rol del Cardenal de Brandsma quien después de la invasión del 10 de Mayo de 1940 toma decisiones sobre una posición clara contra la política de prensa de las autoridades alemanas y sus colaboradores holandeses. Es así que periodístas y directores de periódicos católicos desarrol- 
lan, algunas veces con el apoyo abierto otácito de los consejeros eclesiásticos, prácticas y vistas divergentes, con el riesgo de ir cada vez más comprometiendose. Según la opinión de Brandsma esta actitud peligrosa les llevaba a comprometerse ellos mismos. El logró convencer a los Obispos holandeses, al final del an̂o 1941 , de rechazar absolutamente nuevas medidas de las fuerzas ocupantes que trataron de usar la prensa para propagar la ideología nacional-socialista. Brandsma no argumentó sobre base de una pequen̂a incidencia de la ley eclesiástica o sobre bases constitucionales; él dió a la discusión fundamental acerca de la libertad y responsabilidad de la prensa, una nueva dimensión ética religiosa. Haciendo ésto él estaba dando un mensaje para nuestro tiempo. Siendo también un profeta de la paz, él siempre estuvo predicando y solicitando, aún antes du su martirio, la reconciliación entre los pueblos de Alemania y Holanda, ambos liberados del Nazismo. 